\title{
Editorial: Bioadhesion
}

\author{
Stanislav N. Gorb ${ }^{1 * t}$ and Ken Nakano ${ }^{2 * t}$ \\ ${ }^{1}$ Department of Functional Morphology and Biomechanics, Kiel University, Kiel, Germany, ${ }^{2}$ Faculty of Environment and \\ Information Sciences, Yokohama National University, Yokohama, Japan
}

Keywords: biological surfaces, bioinspired materials, biomimetic systems, attachment, detachment

\section{Editorial on the Research Topic}

\section{Bioadhesion}

Materials and systems preventing the separation of two surfaces may be defined as adhesives. There are a variety of natural adhesive devices based on entirely mechanical principles, while others additionally rely on the chemistry of polymers and colloids. Adhesive organs are functional systems, the purpose of which is either temporary or permanent attachment of an organism to the substrate surface, to another organism, or temporary interconnection of body parts within an organism. Their design varies enormously and is subject to different functional loads. There is no doubt that many functional solutions have evolved independently in different lineages of organisms. Many species of animals and plants are supplied with diverse adhesive surfaces, the morphology of which depends on the species biology, and the particular function in which the adhesive device is involved.

There are numerous publications on cell adhesion phenomena, but much fewer references are devoted to the non-specific adhesion of living organisms. Because of the structural, mechanical, and chemical complexity of biological surfaces related to adhesion, exact working mechanisms have been clarified only for some systems. In this Research Topic (RT) "Bioadhesion", we aimed at collecting articles dealing with biological surfaces and systems specialized for adhesion enhancement. These contributions discuss adhesive function of biological surfaces and their relationship with the structure, contact mechanics and chemistry of surfaces. Because of the diversity of functions in adhesion-related biological surfaces, biology could provide interesting inspirations for a broad range of topics in physics, chemistry, and engineering.

In the following, we briefly introduce the 13 articles comprising this RT. They can be broadly divided into four categories: 1) bacterial adhesion, 2) plant adhesion, 3) animal adhesion, and 4) biomimetic adhesion.

\section{BACTERIAL ADHESION}

This collection of articles begins with a Mini Review article on bacterial adhesion entitled "Modeling Bacterial Adhesion to Unconditioned Abiotic Surfaces" presented by Spengler et al. As a first step toward biofilm formation, understanding bacterial adhesion is of fundamental interests in many applications. The authors presented the approaches of understanding bacterial adhesion on the whole-cell level in the framework of colloidal science and contact mechanics. Then, they discussed the prospects and limitations of the models and describe the efforts made outside the frameworks in describing bacterial adhesion mediated by cell surface macromolecules.

Received: 13 July 2021

Accepted: 26 July 2021

Published: 11 August 2021

Citation:

Gorb SN and Nakano K (2021) Editorial: Bioadhesion.

Front. Mech. Eng 7:740496. doi: 10.3389/fmech.2021.740496

\section{PLANT ADHESION}

Two Original Research articles on plant adhesion are contained in this collection. The first article is "Root Hair Adhesion in Posidonia oceanica (L.) Delile Seedlings: A Numerical Modelling Approach" 
presented by Zenone et al. In the marine environment, the seagrass Posidonia oceanica attaches firmly to solid substrates via adhesive root hairs, forming micro-pad structures at their tips. The authors used novel morphological and ultrastructural data to develop a numerical model to study the dynamics of root hair adhesion during contact formation on rough solid substrates.

The second article on plant adhesion is "Push and Pull: Biomechanics of the Pollination Apparatus of Oncidium spp." presented by Thielen et al. In orchid pollination process, a mechanical barrier ensuring that only suitable pollinators can access the flowers and remove the pollen is the vital selective trait. The authors described the functional morphology of the pollination apparatus in two orchid species, Oncidium wentworthianum and O. otogaya, by experimentally studying adhesive events in the pollination process.

\section{ANIMAL ADHESION}

Six Original Research articles on animal adhesion are contained in this collection. The first article is "Setal Field Transects, Evolutionary Transitions and Gecko-Anole Convergence Provide Insights Into the Fundamentals of Form and Function of the Digital Adhesive System of Lizards" presented by Russell and Garner. During the last two decades many investigations examining the gecko adhesive system have been carried out, where most of the research has been focused on few species. The authors of this broad comparative study examined in detail setal transects of adhesive pads, evolutionary transitions from non-adhesive to adhesive digits, and the convergent evolution of adhesive apparatuses in geckos' and anoles.

The second article on animal adhesion is "Adhesion Behaviors of Abalone Under the Action of Water Flow" presented by Zhang et al. The abalone is well known for its non-permanent adhesive system, detailed information of which might be beneficial to such areas as underwater robotics, high precision sensors, and intelligent devices. The authors conducted experiments, to observe the posture response of an abalone under various water flow conditions. They also performed numerical simulations to display the stress field, reasonably explaining the results of experimental observations.

The third article on animal adhesion is "Adhesive Behavior of Propolis on Different Substrates" presented by Saccardi et al. Propolis is a sticky substance used by bees to seal gaps in their hive and protect the colony against pathogens. The authors performed 1) adhesion tests with propolis on various substrates, 2) differential scanning calorimetry analysis, and 3) compression tests. The obtained data are interpreted from a biomechanical point of view, and the significance of the obtained results for bee biology was discussed.

The fourth article on animal adhesion is "Adhesive Droplets of Glowworm Snares (Keroplatidae: Arachnocampa spp.) Are a Complex Mix of Organic Compounds" presented by Wolff et al. Glowworms are an iconic part of the fauna of Australia and New Zealand that combine the construction of a sticky snare with a bioluminescent lure. The authors studied the chemical composition of the water-soluble fraction of adhesive droplets from the snares of three glowworm species using nuclear magnetic resonance and mass spectrometry. The obtained results contribute to our understanding of the unique prey capture strategy of glowworms.

The fifth article on animal adhesion is "Perspective for a New Bioinspired Permanent Adhesive for dry Conditions - Insights in the Glue Producing Japanese art of Defence System of the Oita Salamander Hynobius dunni" presented by Von Byern et al. The defense secretion system in amphibians is expected to provide potential for novel fast-curing secretion able to adhere to various surfaces under dry conditions. With the microanatomical and histochemical characterization of the endemic Japanese Oita salamander Hynobius dunni, the authors discussed the nature of the chemical composition of glue-producing glands and its divergence to the other well-characterized species Plethodon shermani.

The sixth and final article on animal adhesion is "Adhesion of Individual Attachment Setae of the Spider Cupiennius salei to Substrates With Different Roughness and Surface Energy" presented by Poerschke et al. Dynamic adhesion is known to be a key ability of spiders to climb up smooth surfaces. The authors examined the adhesion of single setae of the spider Cupiennius salei and studied the pretarsus morphology and the fine structure of individual setae. They showed that the adhesion force on the smooth hydrophobic polytetrafluoroethylene was 30\% of that on a smooth hydrophilic glass substrate. The main finding of this article is that both the structure and adhesive performance of individual setae very strongly differ depending on the setal position on the pretarsus.

\section{BIOMIMETIC ADHESION}

Four articles on biomimetic adhesion are contained in this collection: two Review articles are followed by two Original Research articles. The first article is "Applications of Bioinspired Reversible Dry and Wet Adhesives: A Review" presented by Kang et al. Bioinspired adhesives that emulate the unique dry and wet adhesion mechanisms of living systems have been actively explored over the past two decades. This review summarizes recent efforts to apply synthetic dry and wet adhesives, mainly focusing on grippers, robots, and wearable sensors.

The second article on biomimetic adhesion is "Mechanics of Crater-Enabled Soft Dry Adhesives: A Review" presented by Wang et al. Recently, cratered surfaces emerged as a different tool for dry adhesion, as they exhibit many advantageous properties, such as tunable pressure-sensitive adhesion, high underwater adhesive strength, and good reusability. This review summarizes the authors' recent work (combining experimental, modeling, and computational components) on the mechanical characterization of cratered surfaces.

The third article on biomimetic adhesion is "On the Nanomechanical and Viscoelastic Properties of Coatings Made of Recombinant Sea Star Adhesive Proteins" presented by Lefevre et al. To attach to surfaces in the sea, sea stars produce proteinaceous adhesive secretions. Some recombinant proteins adsorb seawater and form coatings on different surfaces. The authors used atomic force microscopy to characterize the nanomechanical properties of 
these coatings with an emphasis on functional characteristics, such as adhesive properties and modulus of elasticity.

The fourth and final article on biomimetic adhesion is "MultiTechnique Investigation of a Biomimetic Insect Tarsal Adhesive Fluid" presented by Fowler et al. The authors combined sum frequency generation with multiple bulk characterization techniques to examine a biomimetic adhesive fluid inspired by the tarsal fluid of insects. They showed that the adhesive mechanism of insect tarsal fluid relies upon contributions from surface-specific properties optimizing traction force and bulk properties promoting rapid surface wetting and maintaining pull-off force for fast detachment.

At the end of this Editorial, the editors would like to express their sincere thanks to all authors for their valuable contributions to this article collection on biological and bioinspired adhesion. It successfully demonstrates how diverse and expansive this field currently is. The editors also strongly hope that the readers will find different perspectives and deep insights in this collection to inspire their future studies on bioadhesion.

\section{AUTHOR CONTRIBUTIONS}

All authors listed have made a substantial, direct, and intellectual contribution to the work and approved it for publication.

Conflict of Interest: The authors declare that the research was conducted in the absence of any commercial or financial relationships that could be construed as a potential conflict of interest.

Publisher's Note: All claims expressed in this article are solely those of the authors and do not necessarily represent those of their affiliated organizations, or those of the publisher, the editors and the reviewers. Any product that may be evaluated in this article, or claim that may be made by its manufacturer, is not guaranteed or endorsed by the publisher.

Copyright (C) 2021 Gorb and Nakano. This is an open-access article distributed under the terms of the Creative Commons Attribution License (CC BY). The use, distribution or reproduction in other forums is permitted, provided the original author(s) and the copyright owner(s) are credited and that the original publication in this journal is cited, in accordance with accepted academic practice. No use, distribution or reproduction is permitted which does not comply with these terms. 